Ann. Sci. forest., 1978, 35 (4), 265-284.

\title{
Contribution à l'étude de la variabilité géographique des Cèdres
}

\author{
M. ARBEZ, P. FERRANDES * ef N. UYAR ** \\ avec la collaboration technique de E. POHOSKI, H. JOLY * et J. POMMERY * \\ Laboratoire d'Amélioration des arbres forestiers I.N.R.A., \\ Pierroton, 33610 Cestas \\ * Station expérimentale de la forêt des Maures, I.N.R.A., \\ Le Ruscas RN 98, 83230 Bormes \\ ** Stagiaire C.I.E.S., \\ Institut des graines forestières et de l'Amélioration, \\ P.K. 11 Gazi/Ankara, Turquie
}

\begin{abstract}
Résumé
Outre la confirmation de caractères de valeur taxinomique déjà connue, l'étude biométriqu de la morphologie des aiguilles et du rameau de jeunes arbres échantillonnés en plantation comparative a permis de révéler de nouvelles variations géographiques existant au sein du genre Cedrus. Ainsi le caractère parfaitement glabre du rameau de l'année chez les populations de Cedrus libani de Turquie étudiées ici permet de les différencier de celles du Liban faiblement pubescentes. De même, des différences ont pu être mises en évidence dans l'espèce Cedrus atlantica : entre les populations algériennes de la zone des Aurès, celles du Rif et du Haut Atlas marocain (longueur de la partie cornée de l'apex des aiguilles, nombre d'aiguilles par rosette, nombre de lignes de stomates, pubescence du rameau). Certains de ces caractères permettent de confirmer l'origine algérienne des Cèdres du Mont Ventoux.

La méthode d'analyse multivariable utilisée pour regrouper objectivement les populations morphologiquement semblables était celle de l'analyse factorielle discriminante.
\end{abstract}

Mots clefs: Genre Cedrus, taxinomie expérimentale (morphologie comparée), variabilité intraspécifique.

\section{1. - Introduction}

Le genre Cedrus est représenté par quatre espèces: deux au Proche Orient (Cedrus libani Barr. en Turquie, en Syrie et au Liban et Cedrus brevifolia Dode. à Chypre) une en Afrique du Nord (Cedrus atlantica Manet. au Maroc et en Algérie) et une dans l'Himalaya (Cedrus deodara Loud.) en Afghanistan et sur le versant sud de l'Himalaya occidental. Hormis leur tempérament montagnard commun, elles poussent dans des conditions écologiques variées avec pour corrolaire des caractéristiques d'adaptation probablement très différentes : entre le bassin méditerranéen et l'Himalaya évidemment mais aussi à l'intérieur même de ces deux grandes zones géographiques (vis-à-vis de la pluviométrie le Rif s'oppose aux Aurès algériens et l'Himalaya occidental à l'Afghanistan). L'écologie et la répartition des espèces ont été 


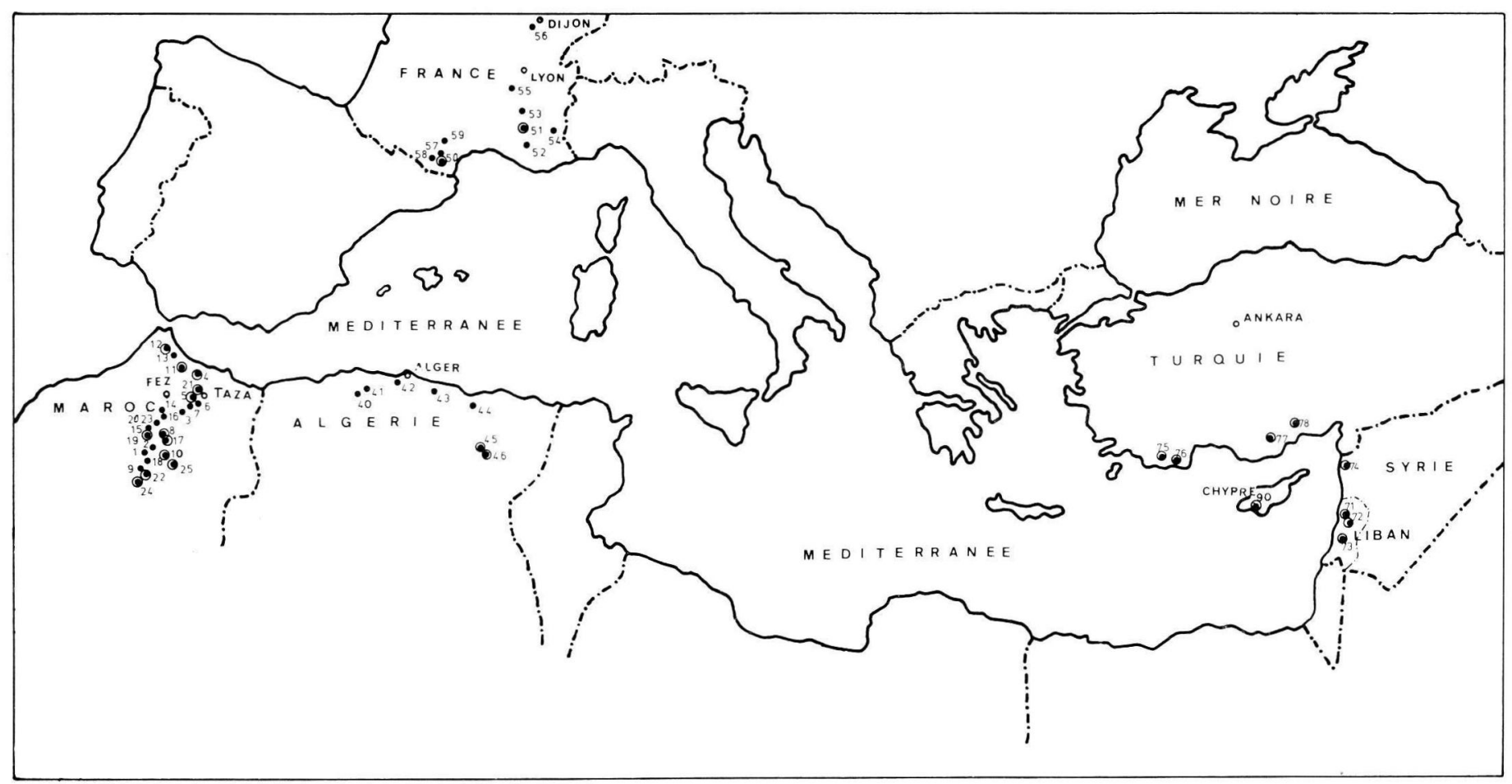

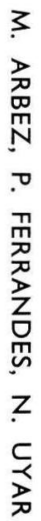

FIG. 1. - Localisation géographique des provenances étudiées (Cedrus deodara excepté).

Geographic localization of the used provenances (Cedrus deodara excepted). 
décrites par divers auteurs, en particulier Emberger (1938), Boudy (1950) Sevim (1955), Basbous ef de Tarade (1955), Millischer (1972), Destremau (1974).

Après le succès des introductions de Cedrus atlantica dans le Ventoux au siècle dernier, cette espèce jouit présentement d'un regain d'intérêt dans les reboisements méditerranéens de l'étage du chêne pubescent. Cela se justifie amplement par ses nombreuses qualités forestières: faible susceptibilité à l'incendie, régénération naturelle possible des peuplements (pérennité des boisements) rusticité, plasticité et croissance initiale supérieure à celles des sapins méditerranéens. Dans ce contexte, des recherches sur la variabilité géographique du genre Cedrus ont été engagées voici 5 ans par I'I.N.R.A. *. Elles reposent sur un réseau de plantations comparatives intéressant désormais neuf stations et environ cinquante provenances différentes représentant l'ensemble de l'aire naturelle.

Avant que ces plantations soient suffisamment âgées pour fournir des informations fiables sur les caractéristiques de croissance et d'adaptation, il semblait important de se faire une idée objective des différences ou des ressemblances morphologiques entre les diverses provenances. De telles informations contribuent incontestablement à la connaissance génétique d'une espèce ou du genre et permettent d'asseoir la systématique sur des bases expérimentales. Plus concrètement, la mise en évidence de caractères spécifiques ou variétaux permet de procéder à des discriminations rigoureuses en pépinière et d'éviter ainsi des erreurs facheuses quant à l'identité du matériel utilisé en reboisement.

\section{1. - Matériel et méthodes}

\section{1. - Echantillonnage des provenances}

Les provenances étudiées ici ne correspondent qu'à une fraction de la collection utilisée pour l'installation des plantations comparatives multistationnelles. Les diverses parties de l'aire naturelle s'y trouvent très inégalement représentées. A titre d'exemple, les provenances marocaines de Cedrus atlantica sont en nombre suffisant pour bien caractériser cette région et mettre en évidence d'éventuelles variations internes, il n'en va malheureusement pas de même des provenances algériennes. Par ailleurs, la partie orientale de l'aire méditerranéenne occupée par Cedrus libani et Cedrus brevifolia est proportionnellement sous-représentée par rapport à la partie occidentale. Quant à Cedrus deodara utilisé comme référence, il ne compte ici que deux provenances originaires de la même région d'Afghanistan.

Même très imparfait, cet échantillonnage n'a pu être rassemblé qu'en faisant appel à trois tranches expérimentales d'âge différent, l'une constituée de semis de 1 an en pépinière du Ruscas (Maures), les deux autres âgées respectivement de 4 et 2 ans après plantation sur une autre station forestière des Maures (Le Treps, Forêt domaniale de Collobrières).

Cette fragmentation de l'échantillon a obligé à des calculs et des interprétations séparées. Le tableau 1 et la figure 1 permettent de situer géographiquement les provenances de la zone méditerranéenne.

\footnotetext{
* Elles ont bénéficié de la collaboration de l'Institut de Recherches forestières de Rabat pour la prospection et la récolte des provenances marocaines. Ces recherches ont été financées avec l'aide de la D.G.R.S.T. et d'un fond de concours du Service des forêts du Ministère de l'Agriculture, consacré aux recherches sur la protection et la reconstitution de la forêt méditerranéenne.
} 
TABLEAU 1

Description des provenances utilisées

Description of provenances

\begin{tabular}{|c|c|c|c|c|c|c|c|c|}
\hline Expérience & Espèce & Pays & Région & Abrev. & Nom & No & Altitude & Origine \\
\hline $\begin{array}{c}1.153 \\
\text { (1 an, pépinière) }\end{array}$ & 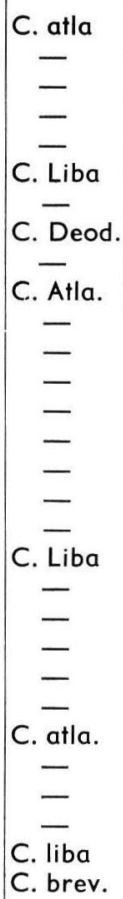 & $\begin{array}{l}\text { Maroc } \\
- \\
\text { Algérie } \\
- \\
\text { Turquie } \\
- \\
\text { Afghanistan } \\
- \\
\text { France } \\
\text { Maroc } \\
- \\
- \\
- \\
- \\
- \\
- \\
\text { France } \\
\text { Turquie } \\
- \\
- \\
\text { Liban } \\
- \\
\text { Maroc } \\
- \\
\text { - } \\
\text { France } \\
\text { Liban } \\
\text { Chypre }\end{array}$ & \begin{tabular}{|l|} 
Taza \\
Haut Atlas \\
Aurès \\
Aurès \\
Taurus orient. \\
Taurus occid. \\
Forêt de Kotgai \\
\multicolumn{1}{|c|}{-} \\
Mont Ventoux \\
Rif \\
Rif \\
Rif \\
Taza \\
Moyen Atlas \\
Haut Atlas \\
Haut Atlas \\
Mont Ventoux \\
Taurus orient. \\
Taurus occid. \\
Taurus orient. \\
Haut Liban \\
Haut-Liban \\
Haut Liban \\
Taza \\
Moyen Atlas \\
Moyen Atlas \\
Corbières \\
Haut Liban
\end{tabular} & $\begin{array}{l}\text { TAZE I } \\
\text { TIMA } \\
\text { MAOU } \\
\text { CHEL } \\
\text { REFA } \\
\text { POZA } \\
\text { KATR } \\
\text { AFGH I } \\
\text { AFGH II } \\
\text { BEDO } \\
\text { KELT } \\
\text { IFRI } \\
\text { IKAN } \\
\text { TAZE II } \\
\text { TAGA } \\
\text { TOUN } \\
\text { ASSA } \\
\text { VENT } \\
\text { POZA } \\
\text { SUTL } \\
\text { ASLA } \\
\text { BECH } \\
\text { HA-AK } \\
\text { HA-JI } \\
\text { TAZE I } \\
\text { IJDR } \\
\text { CZAD } \\
\text { RIAL } \\
\text { BARO } \\
\text { PAPH }\end{array}$ & $\begin{array}{l}\text { Tazekka } \\
\text { Tizi Makran } \\
\text { Maoutfond } \\
\text { Chelia } \\
\text { Refaa (Belezma) } \\
\text { Pozanti } \\
\text { Katrandag } \\
\text { Afghanistan I } \\
\text { Afghanistan II } \\
\text { Bedouin } \\
\text { Jbel Kelti } \\
\text { Tizi Ifri } \\
\text { Ikankaben } \\
\text { Tazekka II } \\
\text { Ta tgaline } \\
\text { Tounfite } \\
\text { Assaka } \\
\text { Mont Ventoux } \\
\text { Pozanti } \\
\text { Sutlegen } \\
\text { Aslankoy } \\
\text { Becharé } \\
\text { Hadat el Akka } \\
\text { Hadat el Jibbe } \\
\text { Tazekka } \\
\text { ljdrane } \\
\text { Col de Zad } \\
\text { Rialsesse } \\
\text { Barouk } \\
\text { Paphos }\end{array}$ & $\begin{array}{r}51 \\
12 \\
4 \\
11 \\
5 \\
8 \\
22 \\
24 \\
51 \\
78 \\
75 \\
77 \\
72 \\
71 \\
71 \\
21 \\
19 \\
17 \\
50 \\
73 \\
80\end{array}$ & \begin{tabular}{|lll}
1 & $600 \mathrm{~m}$ \\
1 & $700 \mathrm{~m}$ \\
1 & $720 \mathrm{~m}$ \\
1 & $900 \mathrm{~m}$ \\
1 & $800 \mathrm{~m}$ \\
2 & $050 \mathrm{~m}$ \\
& \\
1 & $500 \mathrm{~m}$ \\
1 & $400 \mathrm{~m}$ \\
1 & $800 \mathrm{~m}$
\end{tabular} & $\begin{array}{c}\text { naturelle } \\
- \\
- \\
- \\
- \\
- \\
- \\
\text { artificielle } \\
\text { naturelle } \\
- \\
- \\
- \\
- \\
- \\
\text { artificielle } \\
\text { naturelle } \\
- \\
- \\
\text { naturelle } \\
- \\
- \\
- \\
\text { - } \\
\text { artificielle } \\
\text { naturelle } \\
-\end{array}$ \\
\hline
\end{tabular}

Le nombre moyen d'individus observés par provenance variait suivant l'expérience (pépinière 15, plantation de 2 ans: 12, plantation de 4 ans : 22).

\section{2. - Caractères observés ou mesurés}

En pépinière et sur le terrain de légères différences sont intervenues dans le choix des caractères observés ou mesurés. Le tableau 2 en donne la liste exhaustive et la description. II est complété par les schémas de la figure 2 et les photographies de la figure 3.

\section{3. - Méthodes d'analyse biométrique utilisées}

Pour opérer une classification précise et objective des populations de Cèdre entrant dans le cadre de cette étude, nous étions contraints de recourir à un système d'analyse multivariable. Dans ce but, l'analyse factorielle discriminante recherche les directions de l'espace (axes canoniques) sur lesquelles la séparation des popu- 


\section{TABLEAU 2}

Liste des caractères étudiés

List of measured traits

$$
\text { A. - Pépinière }
$$

\section{Caractère}

1 Longueur des aiguilles de l'auxiblaste (obtenue en faisant la moyenne des longueurs des deux aiguilles opposées, choisies au milieu du rameau de l'année de chaque individu mesuré)

2 Angle d'insertion des aiguilles de l'auxiblaste (obtenu en faisant la moyenne des angles d'insertion des deux aiguilles précédemment mesurées et non pourvues d'un mésoblaste à leur aisselle)

3 Longueur de la partie cornée de l'apex d'une aiguille de l'auxiblaste (mesurée au micromètre occulaire $\times 32$ ).

4 Nombre d'aiguilles d'une rosette. On a choisi une rosette située en deuxième position à partir de l'extrémité du rameau de l'année, les aiguilles courtes formant la rosefte sont insérées sur un rameau demi-court ou mésoblaste

5 Longueur de l'aiguille la plus longue de la rosette précédente

6 Nombre maximum de lignes de stomates sur une face de l'aiguille la plus longue de la rosette précédente

7 Pubescence du rameau de l'année, estimée par le nombre de poils visibles sur trente graduations du micromètre occulaire $(\times 32)$

\section{B. - Plantation comparative}

$\mathrm{n}^{\mathrm{O}}$

\section{Caractère}

Nombre d'aiguilles d'une rosette du rameau de 2 ans (on a choisi cette rosette dans la portion du rameau ne comportant pas d'allongement des mésoblastes)

\section{Longueur de l'aiguille la plus longue de la rosette précédente}

Nombre maximum de lignes de stomates sur une face de l'aiguille la plus longue de la rosefte précédente

Longueur de la partie cornée de l'apex de l'aiguille la plus longue de la rosette précédente (mesurée au Micromètre occulaire $\times 32$ )

Longueur du mésoblaste allongé le plus long $(\mathrm{mm})$

Pubescence du rameau de l'année, estimée par le nombre de poils visibles sur trente graduations du micromètre occuiaire $(\times 32)$

lations (ici, provenances) est la plus efficace. Cette méthode s'appuie sur l'utilisation simultanée des matrices de variance-covariance inter et intra population. Les éclaircissements théoriques nécessaires à sa compréhension se trouvent dans divers ouvrages d'analyse multivariable (en particulier T. W. Andersson, 1958 ; H. Seal, 1964 ; D. F. Morrison, 1967 ; P. Dagnelie, 1973). 


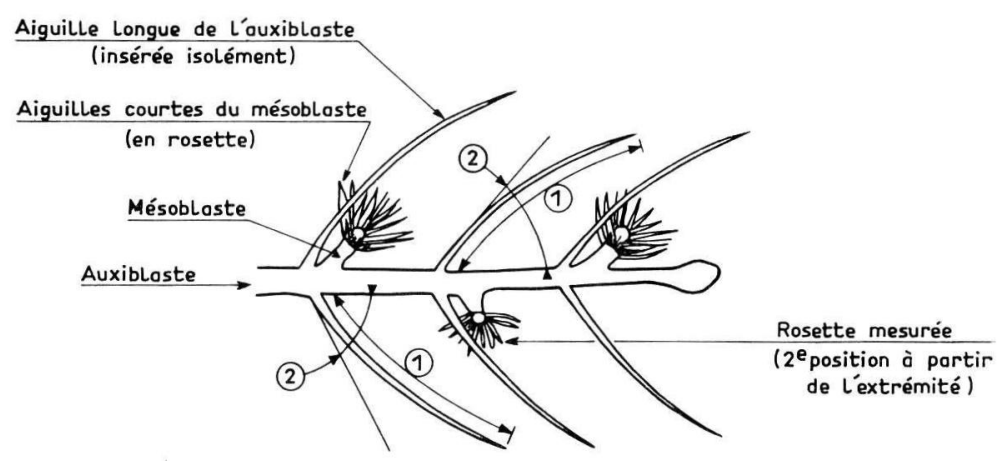

Organisation d'un échantillon de pépinière (rameau d'un an)

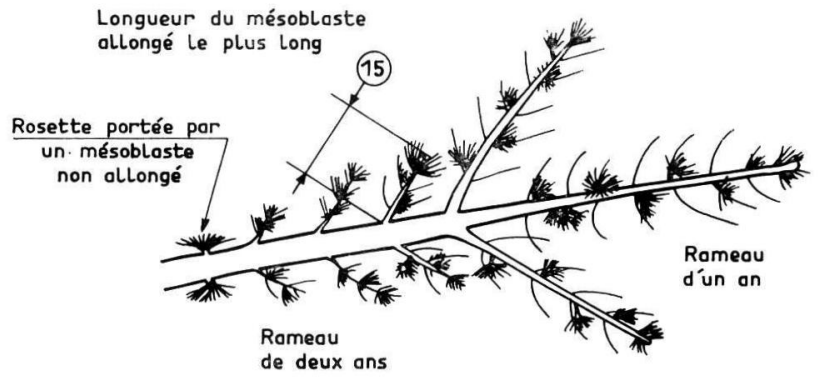

Organisation d'un échantillon de plantotion ( 2 à 4 ons)

FIG. 2. - Description des caractères mesurés.

Description of the measured traits.

Si $B$ et $W$ désignent les matrices des sommes de carrés et de produits centrés «INTER » et «INTRA » provenance, le critère de discrimination $\Phi=\frac{V B V}{V W V}$ atteint son maximum pour $V_{1}$, vecteur propre correspondant à la plus grande valeur propre $\lambda_{1}$ de $(B-\lambda W) V=0$; alors $\Phi=\lambda_{1}$ et $V_{1}$ est dit premier axe canonique. Les axes canoniques suivants correspondent aux valeurs décroissantes successives de $\lambda$. On décide de la dimension de l'espace discriminant compte tenu de la variabilité «INTRA » et on représente les points moyens des provenances dans l'espace des variables initiales réduites par leurs écarts-types «INTRA ». La variabilité «INTRA » vaut alors 1 sur les différents axes canoniques, le graphique est alors isotrope et permet le tracé d'un cercle de confiance autour de chaque point moyen. Le rayon du cercle de confiance est l'inverse de la racine carrée du nombre moyen d'observations par provenance.

La signification biologique des axes canoniques est donnée par l'importance relative des coefficients des diverses variables dans leur équation et par l'étude des corrélations entre varicibles canoniques au sens INTER ef variables initiales. 


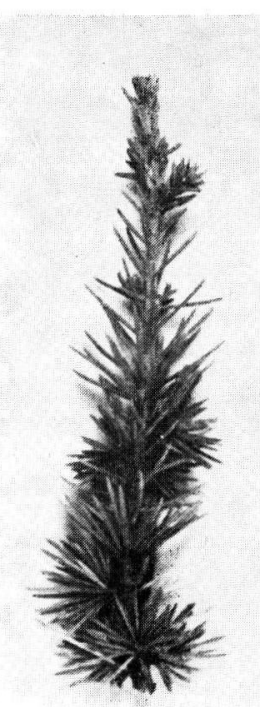

IFRI

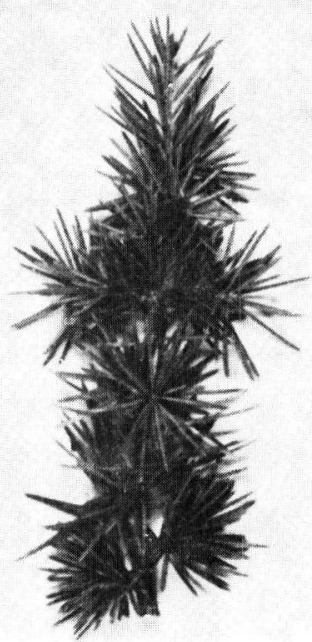

TAZE II

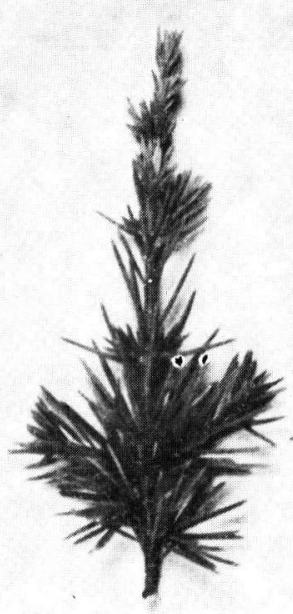

TUUN

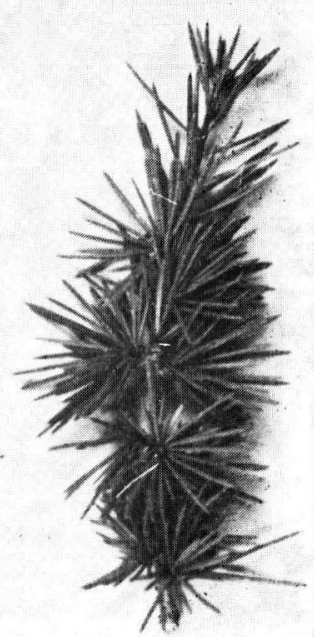

BELE

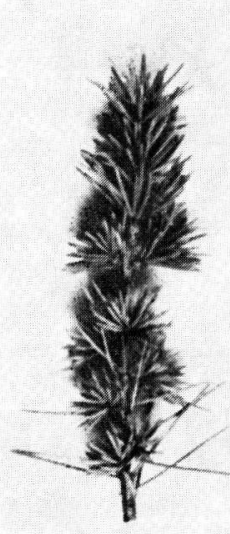

$\mathrm{PAPH}$

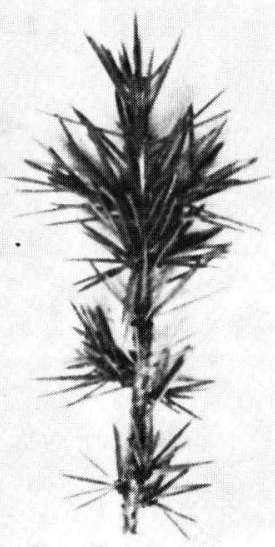

POZA

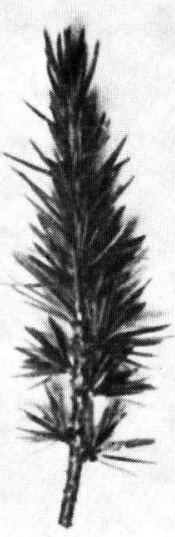

BARO

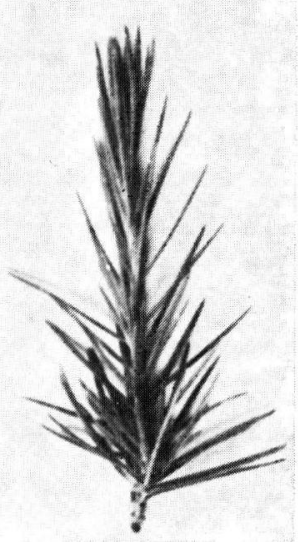

AFGH

\footnotetext{
Groupe occidental : en haut

Groupe oriental : en bas
}

FIG. 3. - Organisation du rameau de Cèdre.

Organization scheme of the Cedrus twig.

Pour ne pas alourdir la lecture de l'article, et parce que la méthodologie biométrique n'était pas l'objectif principal de cette étude nous avons choisi de ne pas reproduire ces tableaux de corrélation *.

* Ils pourront toutefois être communiqués aux lecteurs intéressés sur simple demande. 


\section{2. - Résultats}

\section{1. - Pépinière}

2.11. Analyse multivariable.

L'analyse factorielle discriminante met en évidence quatre axes canoniques significatifs. Nous raisonnerons surtout sur les 3 premiers.

Le premier axe canonique $C_{1}$ dépend directement de la longueur totale des aiguilles de l'auxiblaste et de celle de leur partie cornée, il est affecté en sens opposé par la pubescence du rameau de l'année et le nombre des aiguilles par rosette.

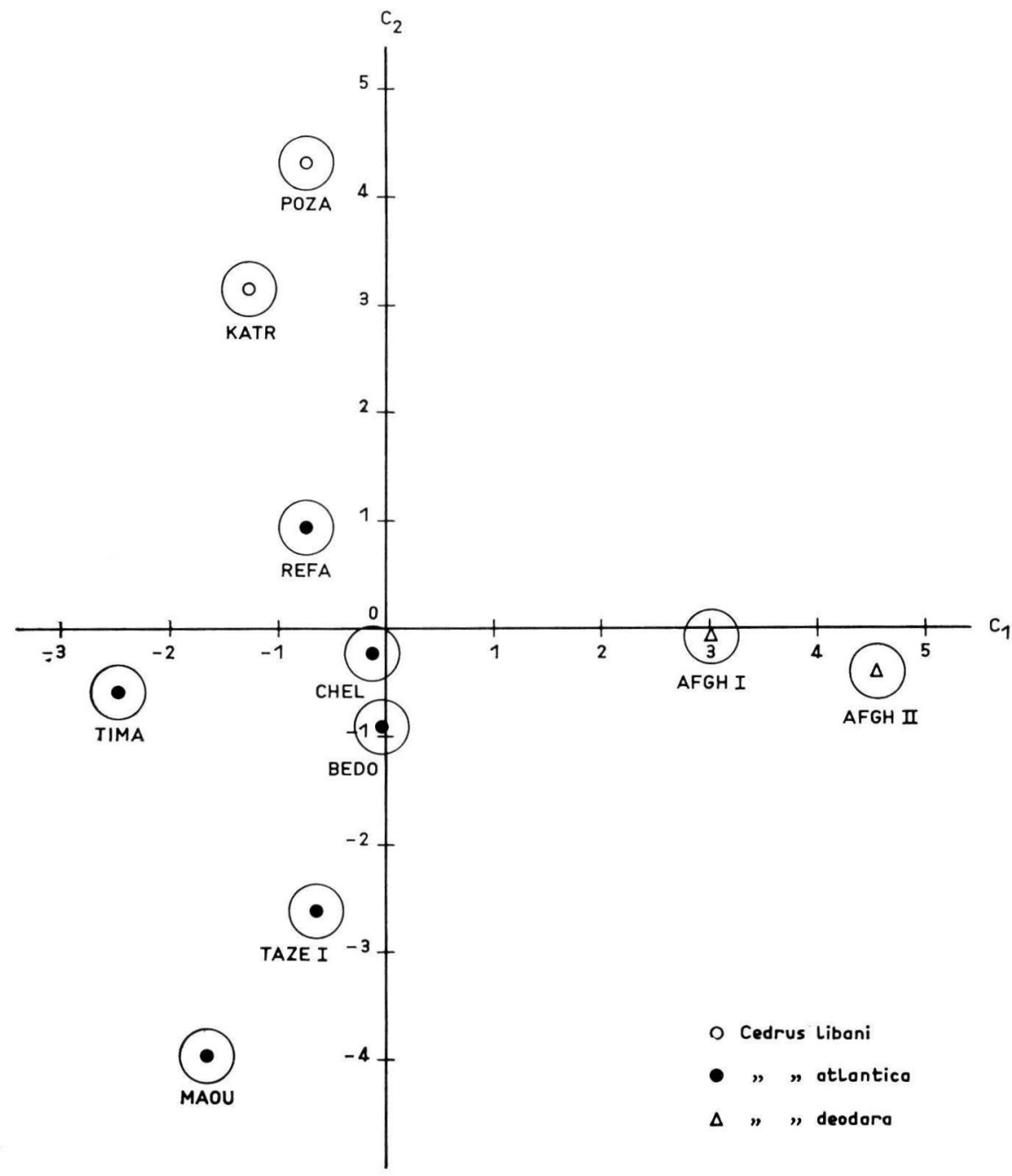

FIG. 4. - Analyse factorielle discriminante 1 (pépinière, semis 1,0).

Projection des points représentatifs des provenances sur le plan défini par les axes canoniques $C_{1}$ et $C_{2}$.

Discriminant factor analysis 1 (Nursery, 1.0 seedlings).

Projection of the points representative of the provenances on the plan definited by the canonical axes $C_{1}$ and $C_{2}$. 
L'axe $C_{2}$ est caractérisé par la prédominance de la longueur des aiguilles de la rosette. $\mathrm{C}_{3}$ dépend négativement du nombre de lignes de stomates ef de la pubescence du rameau de l'année. La figure 4 situe graphiquement les provenances de l'échantillon dans le plan défini par $C_{1}$ et $C_{2}$. Cette représentation dégage 4 groupes géographiques (Turquie, Afghanistan, Algérie, Maroc) dont l'existence s'explique parfaitement en considérant les caractéristiques analytiques des provenances qui les constituent (§ 2.12).

\subsection{Analyses univariables.}

Pour chaque caractère pris séparément, le graphique des moyennes de provenances, le test de Duncan, et le test $F$ de l'analyse de variance à un facteur sont reproduits en figure 5 .

Sur ces jeunes semis d'un an, les aiguilles de l'auxiblaste sont de longueur comparable chez Cedrus libani et Cedrus deodara mais beaucoup plus longuement cornées au niveau de l'apex chez Cedrus libani de Turquie. Les aiguilles insérées en rosette sur le mésoblaste sont nettement plus longues chez le Cèdre Deodar d'Afghanistan. Autre différence permettant de le distinguer: le rameau d'un an est faiblement pubescent alors qu'il est rigoureusement glabre chez les deux provenances de Cedrus libani de Turquie étudiées ici (cf. Pozanti, fig. 6).

Comparées à celles des deux grandes espèces orientales, les aiguilles de l'auxiblaste sont plus courtes chez Cedrus atlantica. Plus, dans l'échantillonnage de provenances utilisé, la longueur diminue notablement d'Algérie au Maroc comme s'il existait un gradient de variation continu de ce caractère d'Est en Ouest de l'aire de répartition du Cèdre. La même observation vaut pour la longueur des aiguilles en rosette insérées sur le mésoblaste. Au Maroc, même la provenance Maoutfond (Hauł Atlas) se caractérise par des aiguilles plus courtes et un plus grand nombre de lignes de stomates. Par compensation et suggérant une répartition différente de la matière sèche, le nombre des aiguilles par rosette varie en sens opposé : il est maximum au Maroc, il diminue en Algérie et atteint les valeurs les plus faibles dans la partie orientale de l'aire (Cedrus libani, Cedrus deodara). On remarque aussi le plus grand nombre des lignes de stomates et l'apex longuement corné des aiguilles de l'auxiblaste des provenances algériennes. Chez de nombreuses espèces de conifères, ces caractères vont souvent de pair avec l'adaptation à la sécheresse (ou à l'altitude). La combinaison de ces deux caractères et d'un angle d'insertion des aiguilles plus ouvert pourrait peut-être expliquer l'aspect bleuté du feuillage des provenances algériennes au stade juvénile.

En passant, il apparaît que la provenance Bedoin (Mont Ventoux) se range dans le groupe formé par les deux provenances algériennes. Cette ressemblance morphologique confirme s'il en était besoin l'origine du plus grand des peuplements artificiels français. On sait en effet qu'il fut créé à partir de 1862 par l'Inspecteur des Forêts Tichadou à partir de graines importées d'Algérie.

Hormis le caractère glabre des deux provenances de Cedrus libani de Turquie, la pubescence du rameau de l'année semble totalement erratique, fluctuant fortement à l'intérieur d'une même région géographique. Sans doute faut-il en chercher l'explication dans la neutralité d'un tel caractère vis-à-vis de la sélection naturelle et l'absence de liaison génétique avec des caractères pourvus d'une valeur adaptative. 


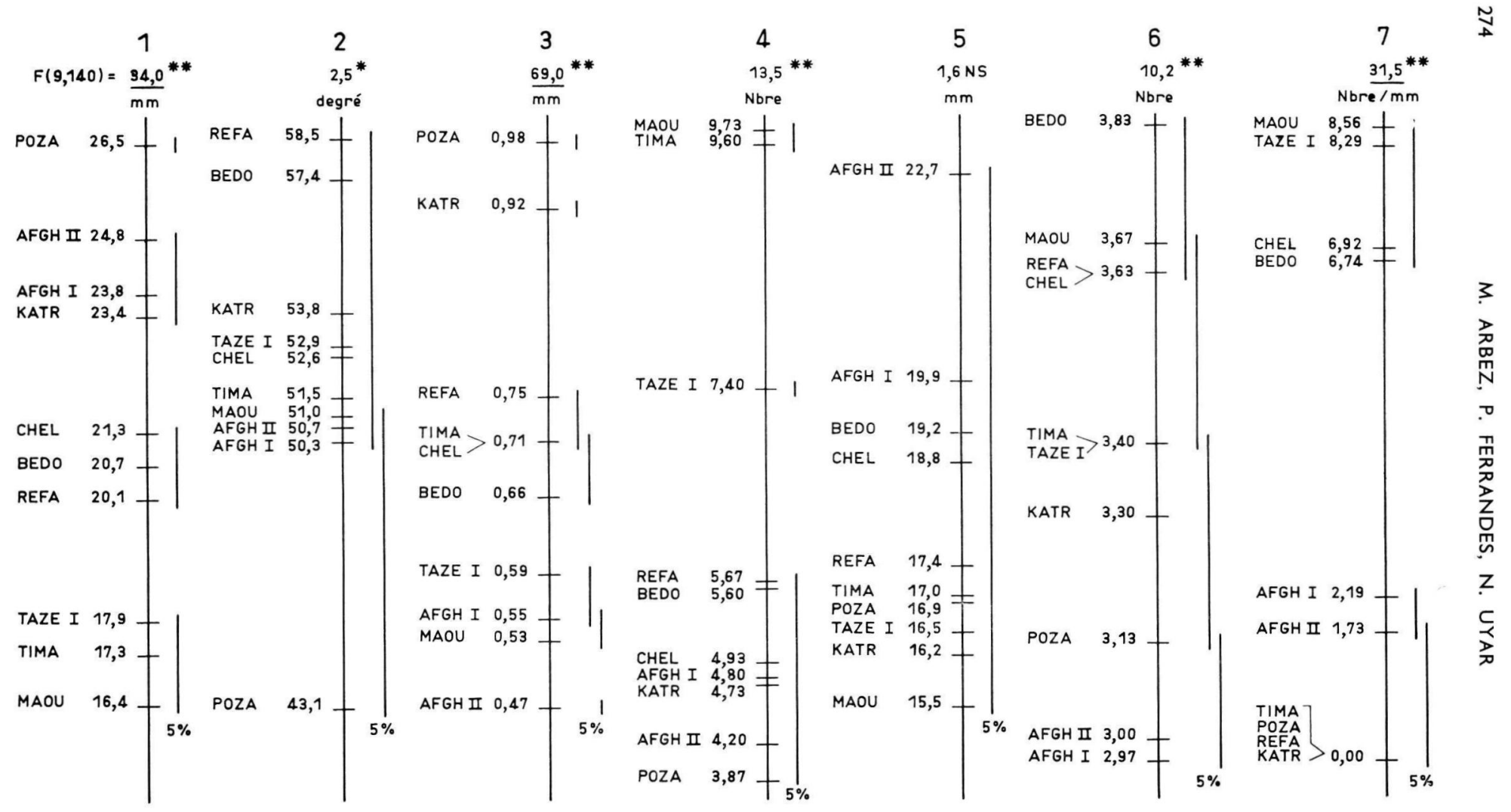

FIG. 5. - Représentation des moyennes de provenances des différents caractères

et de leurs différences significatives (pépinière, semis 1.0).

Representation of the provenance means for the different traits

and of the statistically significant differences (Nursery, 1.0 seedlings). 


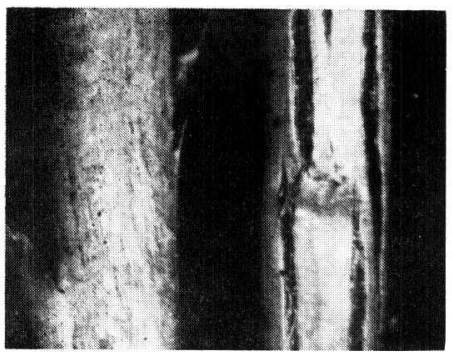

BARO

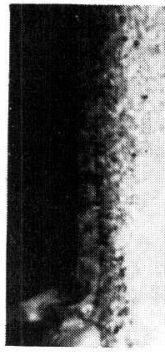

TOUN

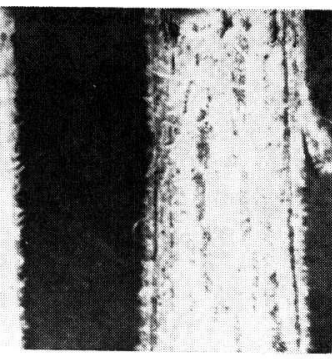

BELE

FIG. 6. - Pubescence du rameau de l'année.

Hairiness of annual twig.

2.2. - Première plantation comparative

(expérience 1.152.3, 2 ans après plantation)

\subsection{Anayse multivariable.}

L'analyse factorielle discriminante montre que les 4 premières valeurs propres sont significatives. Le premier axe canonique $C_{1}$ combine le nombre d'aiguilles par rosette et la pubescence affectés du signe plus avec la longueur de la partie cornée de l'apex affectée du signe moins. L'axe $C_{2}$ est encore marqué par la prédominance de la longueur de la partie cornée de l'apex alliée avec le même signe plus avec la longueur des aiguilles en rosette et l'allongement maximum des mésoblastes du rameau de deux ans. L'axe $C_{3}$ est surtout conditionné négativement par la longueur des aiguilles en rosette et le nombre de lignes de stomates (fig. 7).

La représentation des provenances dans le plan des axes $C_{1}$ et $C_{2}$ sépare progressivement Cedrus atlantica de Cedrus libani du Liban puis de Cedrus libani de Turquie, ce dernier se trouvant finalement très éloigné des deux précédents. La même configuration se retrouve dans le plan $C_{1} C_{3}$. Si l'on se rappelle que les points moyens sont représentés dans une "base canonique réduite intra », la distance généralisée entre deux populations ( $\mathrm{D}^{2}$ de Mahalanobis) est égale à la distance euclidienne séparant les points moyens de ces deux populations. Dans l'espace canonique les populations de cèdre du Liban apparaissent donc morphologiquement plus proches de celles de Cedrus atlantica que les populations très originales de Cedrus libani de Turquie.

En regard de différences aussi marquées, les populations marocaines entre elles (Rif, Moyen Atlas, Haut Atlas) ou le Mont Ventoux d'origine algérienne ne se séparent pas.

\subsection{Analyses univariables.}

La figure 8 donne les moyennes de provenances de chaque caractère, leurs différences significatives (Test de Duncan) et le test $F$ de l'analyse de variance à un facteur. L'originalité des provenances rifaines, non perceptible à l'analyse multivariable, est clairement exprimée par l'allongement maximum du mésoblaste et le nombre des aiguilles par rosette. Au niveau des moyennes de provenances, on note 


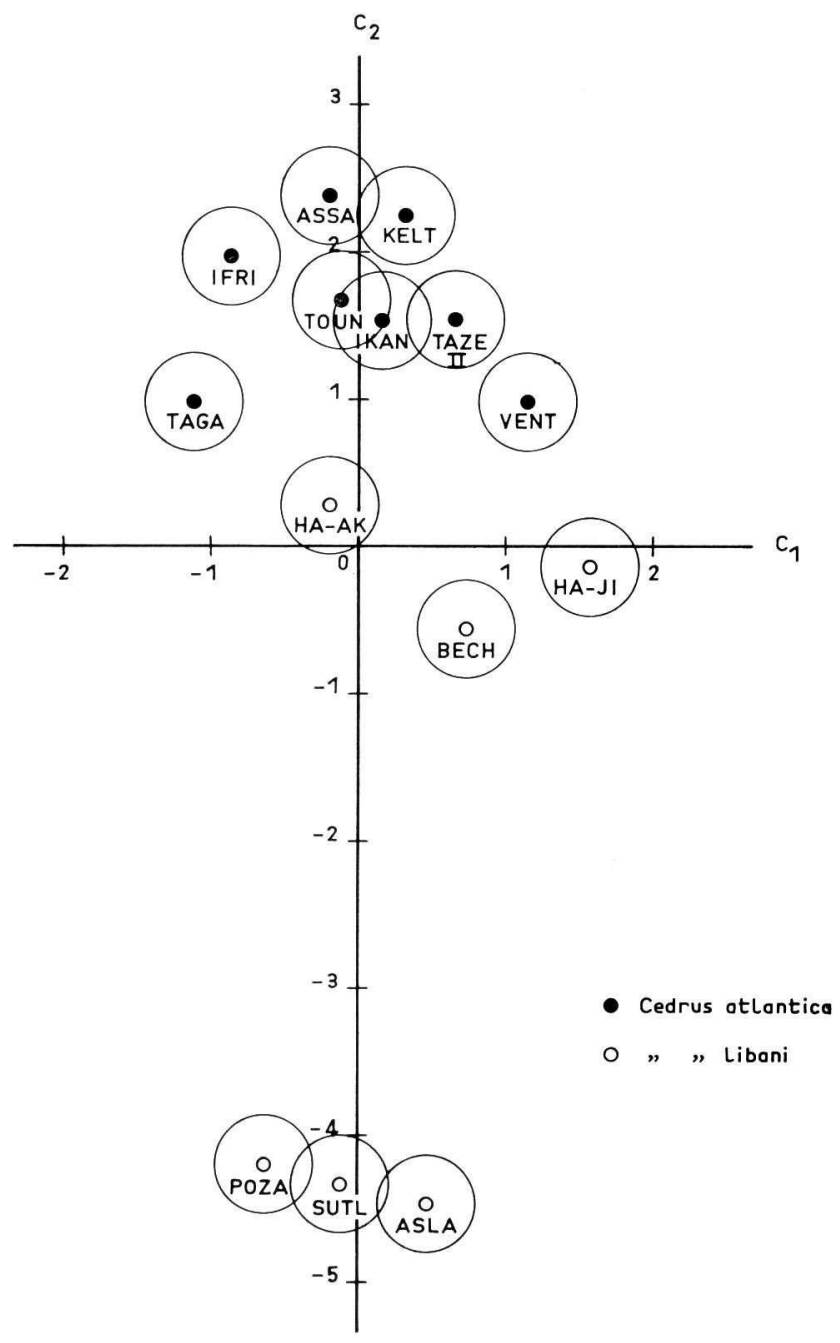

FIG. 7. - Analyse factorielle discriminante 2 (expérience 1.152.3, 2 ans après plantation). Projection des points représentatifs des provenances sur les axes canoniques $C_{1}$ et $C_{2}$.

Discriminant factor analysis 2 (experiment 1.152.3, 2 years after plantation). Projection of the points representative of the provenances on the plan definited by the canonical axes $\mathrm{C}_{1}$ and $\mathrm{C}_{2}$.

d'ailleurs une corrélation compréhensible entre les deux caractères $\left(r=0,7^{* *}\right)$. En fait les provenances du Rif et de la tache de Taza (Tazzeka) sont caractérisées par un fort allongement des mésoblastes en fin de saison de végétation accompagné d'un plus grand nombre d'aiguilles par rosette. Bien que cela soit moins net la longueur des aiguilles en rosette semble plus faible chez les provenances du Rif au sens strict.

Par ailleurs, la provenance du Haut Atlas Assaka se caractérise elle aussi par des aiguilles courtes confirmant ainsi le résultat déjà mis en évidence en pépinière sur Maoutfond. Si Tounfite originaire elle aussi du Haut Atlas ne présente pas la 


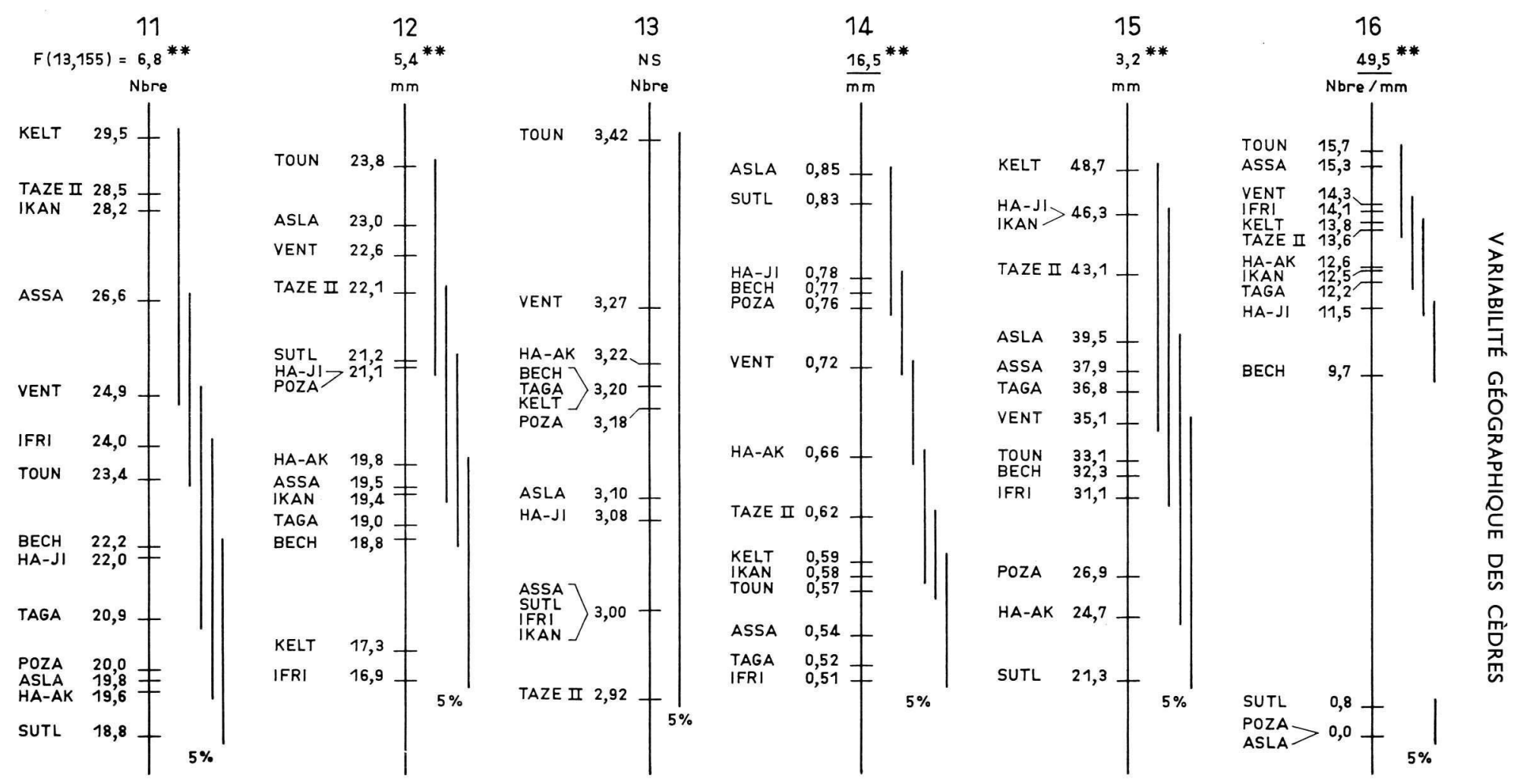

FIG. 8. - Représentation des moyennes de provenances des différents caractères et de leurs différences significatives (expérience 1.152.3, 2 ans après plantation).

Representation of the provenance means for the different traits

and of the statistically significant differences (experiment 1.152.3, 2 years after plantation). 
même particularité, elle compte comme Maoutfond un nombre de lignes de stomates important. Ces trois provenances sont celles qui présentent la pubescence la plus accusée en valeur relative (cf. Tounfite, fig. 6).

Les provenances de Cedrus libani du Liban mais surtout de Turquie possèdent un petit nombre d'aiguilles par rosette et des apex longuement cornés, cette dernière caractéristique est aussi présente à un degré moindre sur les individus d'ascendance algérienne du Mont Ventoux.

Aux différences déjà enregistrées entre Cedrus libani de Turquie et du Liban, s'ajoute le caractère glabre des premières et nettement pubescent des secondes. Pour le reste et à des degrés divers toutes les provenances de Cedrus atlantica du Maroc sont pubescentes.

\section{3. - Seconde plantation comparative}

(expérience $1.150 .2,4$ ans après plantation)

\subsection{Analyse multivariable.}

Les quatre premières valeurs propres sont significatives. L'axe canonique $C_{1}$ compte une participation équilibrée des différents facteurs avec cependant une prédominance de la longueur des aiguilles en rosette, de celle de la partie cornée de l'apex et de la pubescence. Le nombre d'aiguilles par rosette intervient également ainsi que le nombre de lignes de stomates mais en sens opposé.

L'allongement du mésoblaste et le nombre d'aiguilles par rosette avec le signe moins, la longueur de la partie cornée de l'apex avec le signe plus expliquent l'axe $\mathrm{C}_{2}$.

Le nombre de lignes de stomates et la pubescence des rameaux de l'année participent de façon prépondérante à la signification de l'axe $C_{3}$ (signe plus) ainsi que le nombre d'aiguilles par rosette (signe moins). La représentation des provenances dans le plan $C_{1} C_{2}$ (fig. 9) regroupe les 3 provenances marocaines de Cedrus atlantica nettement séparées des provenances orientales de Cedrus libani (Barouk, Liban) et de Cedrus brevifolia (Paphos, Chypre). La provenance artificielle Rialsesse occupe une position intermédiaire mais significativement distincte, entre le groupe des provenances marocaines et Barouk.

\subsection{Analyses univariables.}

Les moyennes de provenances caractère par caractère, leurs différences significatives et le test $\mathrm{F}$ de l'analyse de variance à un facteur sont reproduites en figure 10. Les deux provenances de Méditerranée orientale sont parfaitement originales comparées à leurs homologues de la portion marocaine de l'aire naturelle, les aiguilles très courtes de Paphos confirment l'appellation «brevifolia » donné à l'écotype chypriote. Mais dans cette expérience la longueur des aiguilles du Cedrus libani reste comparable à celle des aiguilles de Cedrus atlantica. Par contre, le petit nombre d'aiguilles par rosette, l'apex longuement corné, et la pubescence plus faible de leur rameau les différencient ensemble des provenances de Cedrus atlantica.

A l'inverse, ces dernières possèdent en commun un fort allongement des mésoblastes traduisant l'existence d'une croissance d'arrière saison. A ce titre, Rialsesse, provenance artificielle se comporte différemment. On remarque enfin qu'ici encore le nombre des aiguilles par rosette semble fortement conditionné par l'allongement 


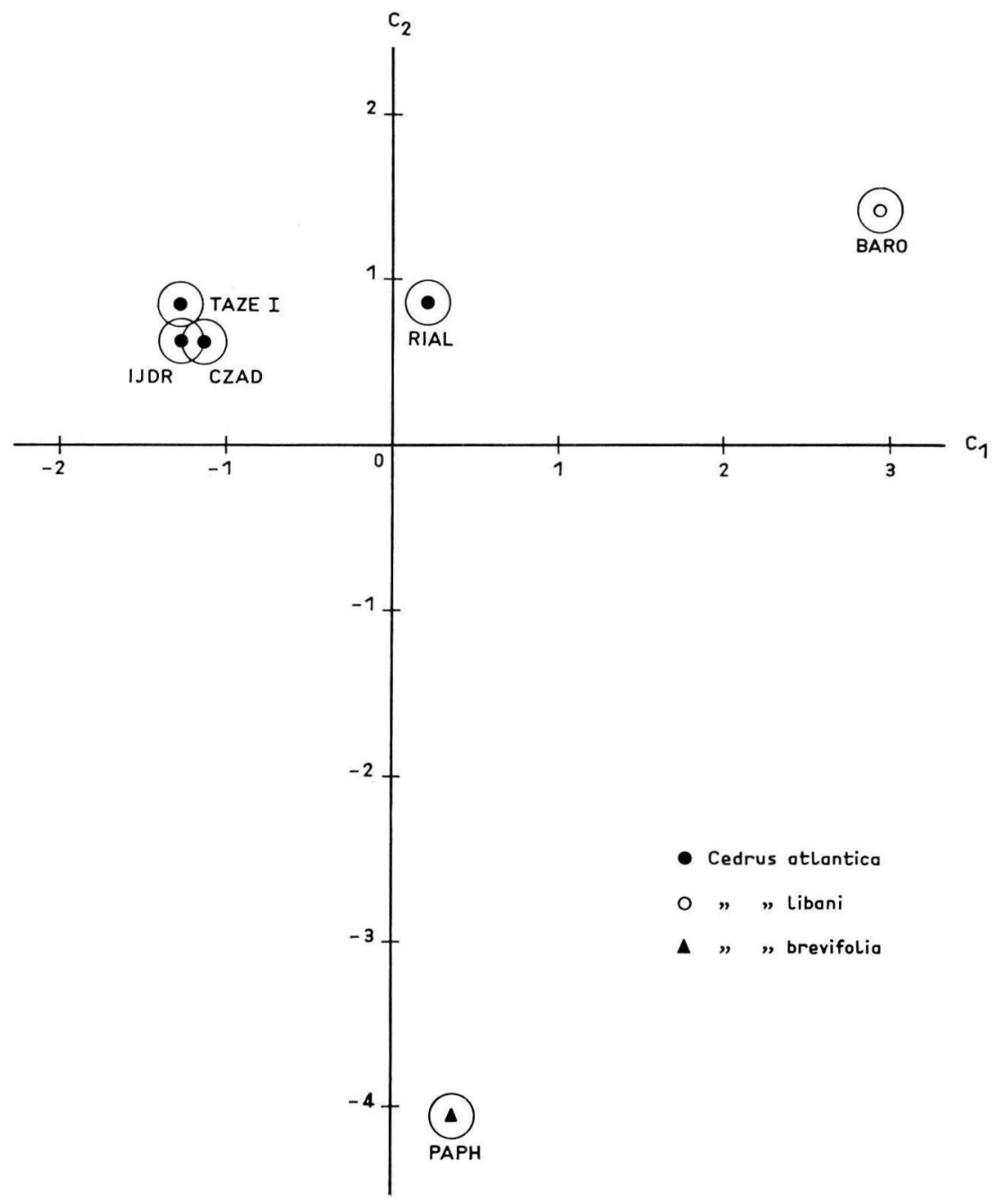

FIG. 9. - Analyse factorielle discriminante 3 (expérience 1.150.2, 4 ans après plantation).

Discriminant factor analysis 3 (experiment 1.150.2, 4 years after plantation).

du mésoblaste en arrière saison, comme en témoigne une excellente corrélation entre les deux caractères ( $r=0,80 * *$ au niveau inter provenances).

\section{3. - Conclusions}

Cette étude préliminaire, engagée sur un échantillonnage de provenances encore restreint et à un stade juvénile, n'en a pas moins permis de dégager quelques caractères distinctifs originaux ef leurs lois de variation géographique. Ces résultats auront besoin d'être confortés par des études ultérieures. D'ores et déjà ils permettent d'élaborer de nouvelles hypothèses de travail et à ce titre de stimuler la réflexion. 
Une récapitulation des principales différences mises en évidence ici figure au tableau 3 et peut servir de base pour l'élaboration de tests variétaux.

TABLEAU 3

Essai de caractérisation des espèces sur la base des caractères morphologiques éfudiés Tentative characterization of the species on the basis of the morphological traits which were studied

\begin{tabular}{|c|c|c|c|c|c|c|}
\hline \multirow[b]{2}{*}{$\begin{array}{l}\text { Espèces } \\
\text { et région d'origine }\end{array}$} & \multicolumn{6}{|c|}{ Caractères étudiés } \\
\hline & $\begin{array}{l}\text { Longueur } \\
\text { des aiguilles }\end{array}$ & $\begin{array}{c}\text { Longueur } \\
\text { de la partie } \\
\text { cornée } \\
\text { de l'apex }\end{array}$ & $\begin{array}{l}\text { Nombre } \\
\text { de lignes } \\
\text { de stomates }\end{array}$ & $\begin{array}{l}\text { Nombre } \\
\text { d'aiguilles } \\
\text { par rosette }\end{array}$ & $\begin{array}{l}\text { Longueur } \\
\text { du } \\
\text { mésoblaste }\end{array}$ & $\begin{array}{l}\text { Pubescence } \\
\text { du } \\
\text { rameau }\end{array}$ \\
\hline \multicolumn{7}{|l|}{$\begin{array}{l}\text { Cedrus atlantica } \\
\text { Maroc }\end{array}$} \\
\hline 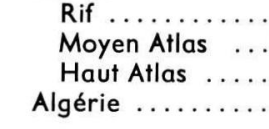 & $\begin{array}{l}x \times x \\
x \times x \times \\
x \times x \\
x \times x \times x\end{array}$ & $\begin{array}{l}x x \\
x x \\
x \\
x x y\end{array}$ & $\begin{array}{l}x \times \\
x \times \\
x \times x \\
x \times x\end{array}$ & $\begin{array}{l}x \times x \times y \\
x \times x \times \\
x \times x \times \\
x \times x\end{array}$ & $\begin{array}{c}x \times x \times \\
x \times x \\
x \times x \\
\text { non éfudiée }\end{array}$ & $\begin{array}{c}x \times x \\
\text { variable } \\
x \times x \times \\
\text { très variable }\end{array}$ \\
\hline $\begin{array}{c}\text { Cedrus brevifolia } \ldots . . . \\
\text { Chypre } \ldots \ldots \ldots\end{array}$ & $x$ & x & $x \times x$ & $x$ & $x \times$ & $x \times$ \\
\hline $\begin{array}{l}\text { Cedrus libani } \\
\text { Liban } \ldots \ldots \ldots \ldots \\
\text { Turquie } \ldots \ldots \ldots \ldots\end{array}$ & $\begin{array}{l}x \times x \times y \\
x \times x \times x \times\end{array}$ & $\left|\begin{array}{l}x \times y \times x \\
x y \times x \times\end{array}\right|$ & $\begin{array}{l}x \times \\
x y\end{array}$ & $\begin{array}{l}x \times x \\
x \times x\end{array}$ & $\underset{x \times}{\text { variable }}$ & 0 (glabre) \\
\hline $\begin{array}{c}\text { Cedrus deodara } \\
\text { Afghanistan }\end{array}$ & $\times \times \times \times \times \times \times$ & $x$ & $x$ & $x \times$ & non étudiée & $y$ (très faible) \\
\hline
\end{tabular}

A côté de la longueur des aiguilles, perceptible de prime abord et déjà utilisée, le nombre d'aiguilles par rosette ef la longueur de la partie cornée de l'apex se sont révélés d'excellents caractères discriminants.

Le Deodar d'Afghanistan se caractérise par des aiguilles très longues, d'un vert assez tendre, souples et à l'apex très peu corné, possédant peu de lignes de stomates. Les rosettes comptent un petit nombre d'aiguilles. La pubescence du rameau est très faible.

Cedrus libani possède encore de grandes aiguilles dotées d'un apex longuement corné, le nombre de ligne de stomates est modéré. Les rosettes possèdent un petit nombre d'aiguilles. Au moins d'après l'échantillonnage de provenances utilisé, le caractère parfaitement glabre du rameau de l'année chez les populations de Turquie permettrait de les différencier clairement de celles du Liban faiblement pubescentes.

Selon G. Callen, le Cèdre du Taurus aurait par ailleurs des aiguilles à face interne glauque et à face externe d'un vert plus clair et lustré comparées à celles du Cèdre du Liban. D'après B. Aytug le pollen de ce dernier serait aussi de dimensions plus faibles que celui des Cèdres du Taurus et souvent malformé ! Enfin à la suite de Gaussen, on peut remarquer que les graines de Cèdres du Liban sont nettement plus grosses que celles du cèdre du Taurus ou de l'Atlas (poids moyen de mille graines calculé sur quatre provenances : $95,5 \mathrm{~g}$ pour le Cèdre du Liban contre 71,2 g pour 

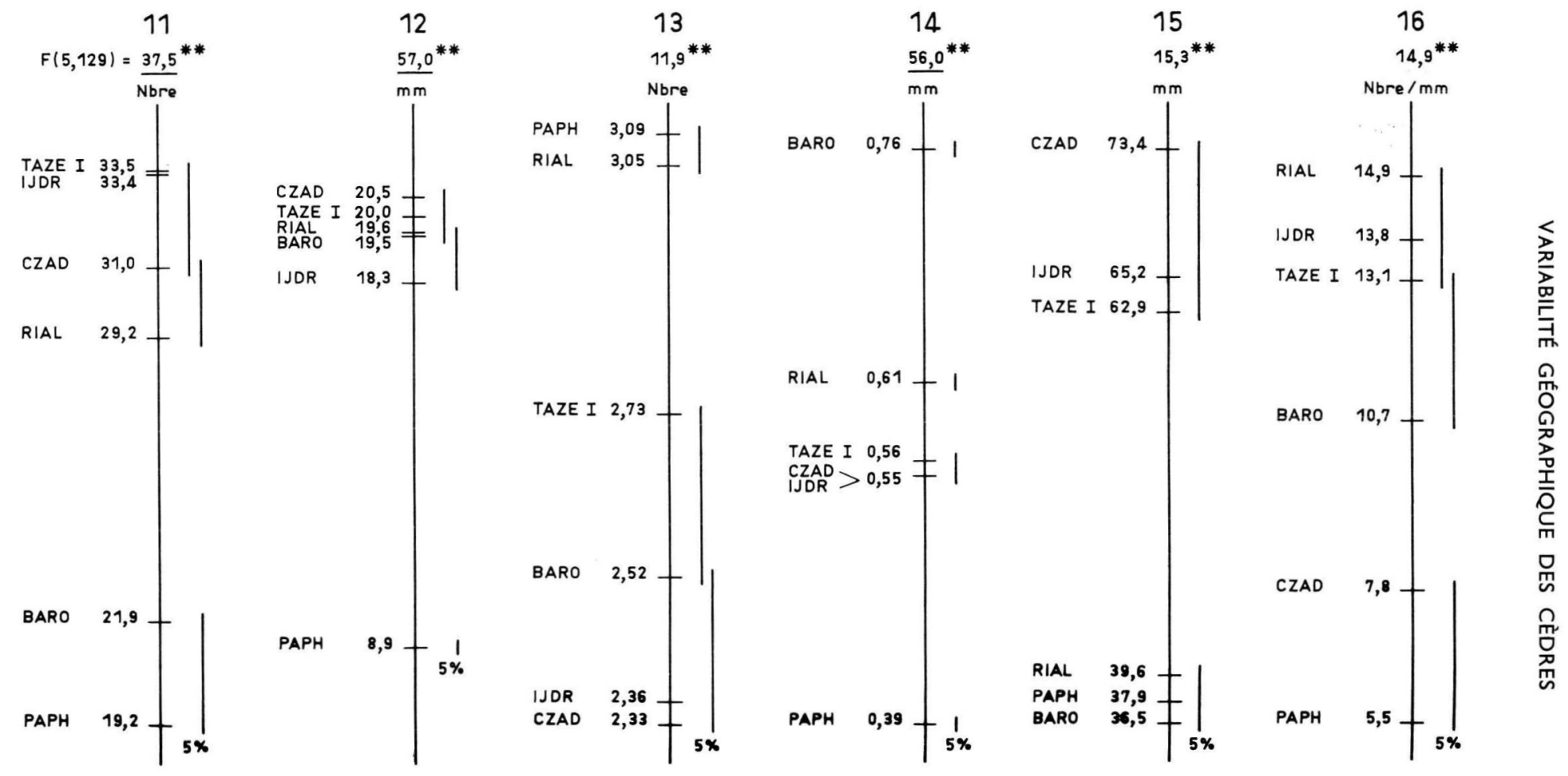

FIG. 10. - Représentation des moyennes de provenances des différents caractères et de leurs différences significatives (expérience 1.150.2, 4 ans après plantation).

Representation of the provenance means for the different traits

and of the statistically significant differences (experiment 1.150.2, 4 years after plantation). 
le cèdre du Taurus et 72,6 g pour quatorze provenances de Cèdre de l'Atlas du Maroc et d'Algérie) *.

Ces différences nombreuses pourraient bien traduire une séparation déjà ancienne des portions turques et libanaises de l'aire naturelle. Alors que les cédraies du Taurus occupent encore aujourd'hui des superficies très importantes, celles du Liban sont réduites à l'état de reliques méridionales. Encore plus restreints, d'autres vestiges subsistent encore dans la zone pontique (Cèdraie de Catalan près de Tokat) ef mériteraient sans doute une étude attentive. Comme le remarque P. Bouvarel, il est possible qu'au cours de périodes anciennes au climat plus humide les sapins aient repoussé le Cèdre dans les régions les plus méridionales, pour autant que la présence continue de chaînes montagneuses suffisamment élevées ait permis cette migration. Grossièrement, l'aire actuelle des cèdres s'appuie en effet sur la limite Sud des Sapins du groupe circum-méditerranéen.

Cedrus brevifolia constitue une entité parfaitement originale caractérisée par de très courtes aiguilles dotées d'un apex brièvement corné et pourvues d'un nombre relativement important de lignes de stomates. Le nombre d'aiguilles par rosette est très petit. La pubescence du rameau de l'année reste faible.

Cedrus atlantica manifeste une variabilité géographique incontestable. Les provenances algériennes étudiées ici, originaires de la zone intérieure de l'aire (Batna) et celles introduites en France au Mont Ventoux se distinguent neittement des provenances marocaines par leurs aiguilles plus longues d'un vert franchement bleuté. Elles possèdent un apex assez longuement corné et de nombreuses lignes de stomates. Elles sont peu nombreuses par rosette. La pubescence du rameau de l'année est très variable.

Des différences existent également à l'intérieur de l'aire marocaine; alors que le Moyen Atlas demeure morphologiquement variable, les deux extrêmes constitués par le Haut Atlas et le Rif sont plus faciles à caractériser. Chez les provenances originaires du Haut Atlas, les aiguilles sont courtes et vert bleuté, avec un apex brièvement corné et de nombreuses lignes de stomates. Le rameau de l'année est très pubescent en valeur relative. Les provenances du Rif possèdent des aiguilles à peine plus courtes que celles du Moyen Atlas et regroupées en grand nombre par rosette. Le rameau de l'année est pubescent. La longueur relative des mésoblastes est importante traduisant l'existence d'un allongement d'arrière-saison.

Indépendamment des caractères signalés ici, G. Callen note que la coupe transversale des aiguilles de $C$. atlantica est aussi large que haute tandis que celle des aiguilles de $C$. libani est plus large que haute.

On peut tenter de donner une interprétation adaptative à certaines particularités morphologiques : bien qu'à peine sensible, le nombre relativement plus important des lignes de stomates chez Cedrus brevifolia et les provenances de Cedrus atlantica des zones les plus continentales d'Algérie (Aurès) et du Maroc (Haut Atlas) coïncide indéniablement avec des conditions d'aridité plus grande. On peut aussi établir un rapprochement entre le nombre d'aiguilles par rosette et la sècheresse de l'aire d'origine, du moins pour les conditions extrêmes caractérisant par exemple le Rif et les Aurès algériens en Méditerranée occidentale, les montagnes du Liban et celles

* Dans les Gymnospermes actuelles et fossiles, Gaussen rapportait des valeurs très voisines : $97 \mathrm{~g}$ pour le Cèdre du Liban et $76 \mathrm{~g}$ pour le Cèdre des Taurus. 
de Chypre en Méditerranée orientale. Cette dernière observation pourrait bien s'appliquer aussi mais en sens opposé à la rapidité de croissance initiale, celle-ci augmentant en moyenne avec le nombre d'aiguilles par rosette de la région d'origine. De telles considérations peuvent apparaître comme momentanément fragiles, elles appellent des vérifications à partir d'échantillonnages de provenances élargis et ultérieurement des tentatives d'explication écophysiologiques. Par quel mécanisme de régulation de sa transpiration l'adaptation à l'aridité du cèdre s'accompagne-t-elle d'une réduction du nombre d'aiguilles par rosette et d'une augmentation du nombre des lignes de stomates?

Reçu pour publication en février 1978.

\section{Remerciements}

Les calculs ont été réalisés par la Station de Biométrie du C.N.R.F.

MM. Debazac, Jacamon (Professeurs à l'Ecole Nationale du Génie Rural des Eaux et des Forêts) et Millier (Directeur de la Station de Biométrie du C.N.R.F.) ont accepté de relire le manuscrit; leurs conseils et leurs critiques ont contribué à sa mise en forme.

Que tous trouvent ici l'expression de nos sincères remerciements.

\section{Summary}

Contribution to the study of the geographic variability of the Genus Cedrus

The biometrical study of morphological characteristics of needles and twigs from young trees sampled in provenance trials, allows to confirm well known taxonomical features and to reveal new geographical variations within the genus Cedrus. Thus the glabrous aspect of the one year old twig appearstypical of the Turkish population of Cedrus libani as compared with those from Lebanon which are thinly pubescent. Besides, differences have been shown within the species Cedrus atlantica, between Algerian populations from the Aures, those from the Rif and those from the Great Maroccan Atlas (length of the hardened part of the apex of needles, number of needles in each set, number of lines of stomates, pubescence of the twig). Some of these characteristics allow to confirm the Algerian origin of the Atlantic Cedar planted on Mont Ventoux (France).

The multivariate statistical analysis used to objectively classify the different populations was the factorial discriminant analysis method.

Key words : Genus Cedrus, experimental taxonomy (compared morphology) intraspecific variability.

\section{Références bibliographiques}

ANDERSON T. W., 1958. Introduction to multivariate statistical Analysis. John Wiley and Sons. AYTUG B., 1961. Etude des pollens du Genre Cèdre (Cedrus Link.). Pollen et Spores, vol. 3, no 1, pp. 47-54.

BASBOUS M., de TARADE R., 1955. Note sur l'écologie du Cèdre du Liban. Université Libanaise, $23 \mathrm{p}$.

BOUDY P., 1950. Economie forestière Nord Africaine. Monographie et traitement des essences forestières, Fasc. 2, pp. 529-619, Ed. Larose.

BOUVAREL P., 1950. Les principales essences forestières du Liban. R.F.F., n ${ }^{0}$ 6, pp. 323-332.

CALLEN G., 1976. Les conifères cultivés en Europe. Vol. 1, 427 p., Ed. Baillière, Paris.

DAGNELIE P., 1973. L'analyse factorielle, in Handbook of vegetation science. Part. 5. Ordination and classification of vegetation. Junk Ed. the Hague.

DEBAZAC E. F., 1964. Manuel des Conifères. Louis Jean, Gap. 1964, pp. 79-82.

DESTREMAU D. X., 1974. Précisions sur les aires naturelles des principaux conifères marocains en vue de l'individualisation des provenances. Annales de la Recherche forestière au Maroc, pp. 5-90. 
EMBERGER L., 1938. Contribution à la connaissance des Cèdres et en particulier du Deodar et du Cèdre de l'Atlas. Revue de Botanique appliquée et d'Agriculture Coloniale, février 1938, vol. 18 , pp. $77-92$.

EMBERGER L., 1938. Les arbres du Maroc et comment les reconnaître. Larose, Paris, 1938,317 p.

GAUSSEN H., 1964. Les gymnospermes actuelles et fossiles. Fasc. 7, Genre Pinus (suite) Cedrus et Abies. Travaux du Laboratoire-forestier de Toulouse, pp. 295-320.

LEPOUTRE B. 1963. Premier essai de synthèse sur le mécanisme de régénération du Cèdre dans le Moyen Atlas Marocain. Annales de la Recherche forestière au Maroc, tome 7, PP. 57-163.

MASSON J. MILLIER, C., GIGOUT M., 1976. Analyse factorielle discriminante. Document à diffusion restreinte 76/007 I. N. R. A. Station deBiométrie du C.N.R.F. Champenoux, 54280 Seichamp.

MILLISCHER H., 1972. Le Cèdre, Nature et forêt Rabat, no 1, pp. 29-46.

MORRISON D. F., 1967. Multivariate statistical method. Mc. Graw Hill Book Company.

ODABACI T., 1967. Recherches sur les graines et les cones des Cèdres du Liban. Revue de la Faculté des Sciences forestière de I'Université d'Istambul, Série A, tome 17, Fasc. 2.

SEAL H., 1964. Multivariate statistical analysis for biologist. Methuen and Co LDT.

SEVIM M., 1955. Répartition et écologie du Cèdre du Liban en Turquie. Publication de la Direction Générale des forêts, n0143, série 24, $98 \mathrm{p}$.

TOTH J., 1970. Plus que centenaire et plein d'avenir : le Cèdre en France. R.F.F., no 3, pp. 355-364. 